\title{
Could Tension between Planck's And Other Observations Be Solved?
}

\author{
Branko M. Novakovic
}

\begin{abstract}
The Hubble constant tension between early and late Universe probes continues to exist. The standard $\Lambda$ cold dark matter ( $\Lambda \mathrm{CDM})$ cosmological model did not solve this tension. Therefore, we must examine potential alternatives to the flat ( $\triangle$ CDM) model that will consistently explain all of the observation data. The related alternative to the theoretical approach could be a new Relativistic Alpha Field Theory (RAFT) that extends GR to the extremely strong gravitational field. The main prediction of RAF theory is a Closed Universe. Using this cosmological model, we calculated the Hubble parameters and compared it with the early (Planck's) and late Universe probes. It is obtained that each probe generates its own cosmological parameters (mass, radius, velocity etc.). Thus, the observation methods used in early and late Universe probes are not equivalent each to the others. Therefore, the tension between early (Planck's) and late Universe probes could not be solved.
\end{abstract}

Index Terms- Relativistic alpha field theory (RAFT), strong gravitational field, tension between early (Planck's) and late Universe probes, this tension could not be solved.

\section{INTRODUCTION}

The recent observations of the Hubble constant $H_{0}$ are discussed in [1]. The results from the Planck observations $[2,3]$ showed the precise constraints on the cosmological parameters to data from CMB observations. Further, relaxing the flat $\Lambda$ CDM model by different complexities [1,4-9] leads to the conclusion that the all presented models could not solve the tension with Planck's observations. Especially, they elaborated the tension in $3.1 \sigma$ with Planck's observations. They concluded that this tension (if unresolved) may force the rejection of the flat $\Lambda \mathrm{CDM}$ model and indicate new physics that will explain our understanding of the cosmology. In the references $[10,11]$ it is also discussed about the possibility that the Planck's evidence for a closed Universe could leads to the possible crisis for cosmology. They concluded that a closed universe can provide a physical explanation of the observation effect with the Planck cosmic microwave background (CMB) spectra. This observation is preferring a positive curvature at more than the $99 \%$ confidence level. They founded that the posterior of the PL18 real temperature and polarization power spectra is centered on a closed model around $\omega_{k}=-0.04$. The prospects for resolving the Hubble constant tension are also discussed in $[12,13,14]$. The most known cosmological model has been developed independently by Alexander Friedmann [15], Georges Lemaitre [16], Howard Percy Robertson [17-19] and Arthur Geoffrey Walker [20]. The Friedman model describes a

Branko Novakovic, FSB - University of Zagreb, Luciceva 5, P.O.B. 509, 10000 Zagreb, Croatia homogeneous, isotropic expanding or contracting Universe. The history and present state of the cosmological models with inclusion of dark matter and dark energy have been presented in [15-38].

Recently, a new approach to the solution of the field equations in a gravitational field is presented in the Relativistic Alpha Field Theory (RAFT) [39-41]. This theory extends GR to the extremely strong gravitational field. The RAF theory is based on the non-vacuum solution of the field equations by including gravitational energy momentum tensor, GEMT, and excluding cosmological constant $\Lambda$. The GEMT is generated in the process of determination of the gravitational field parameters [39-42] instead of adding it by hand on the right side of the field equations, as Einstein suggested. Following the references [39-42], the process of derivation of the new cosmological model has been presented in detail in the reference [43] where it has been theoretically proved that the positive (repulsive) gravitational force could be the source of the dark energy. The repulsive force is produced in the region $G M / 2 c^{2} \leq r<G M / c^{2}$. At the radius $r=G M / 2 c^{2}$ repulsive gravitational force is maximal and at the radius $r=G M / c^{2}$ is equal to zero. Further, in the region $G M / c^{2}<r<\infty$ the gravitational force is attractive. At the radius $r=G M / c^{2}$ gravitational force is changing from repulsive to the attractive one (in the expanding phase) and from attractive to repulsive force (in the contracting phase). Here $G$ is gravitational constant, $M$ is total gravitational mass in Universe, $c$ is a speed of the light in vacuum and $r$ is a gravitational radius.

\section{BRIEF COMPARISON OF FRIEDMAN AND RAFT COSMOLOGICAL MODEL}

Following the assumption that the Universe geometric properties are homogeneous and isotropic, i.e. the Cosmological Principle is valid, Friedman has been derived his equations [15]:

$$
\begin{aligned}
& \left(\frac{\dot{\alpha}}{\alpha}\right)^{2}+\frac{\mathcal{K} c^{2}}{\alpha^{2}}-\frac{\Lambda c^{2}}{3}=\frac{8 \pi G}{3} \rho, \\
& \frac{\ddot{\alpha}}{\alpha}=-\frac{4 \pi G}{3}\left(\rho+\frac{3 p}{c^{2}}\right)+\frac{\Lambda c^{2}}{3} .
\end{aligned}
$$

Here $\alpha(t)$ is the scale factor with related time derivations $\dot{\alpha}$ and $\ddot{\alpha}, \mathcal{K}$ is spatial curvature parameter, $\Lambda$ is cosmological constant, while $\rho$ and $p$ are fluid mass density and pressure, respectively. The presented equations (1) are the basis of the standard big bang cosmological model including the current $\Lambda C D M$ model. New approach to derivation of the cosmological model is based on the new Relativistic Alpha Field Theory (RAFT) presented in [39-41,43]. The RAF theory extends the application of the standard GRT to the extremally strong gravitational field. In this theory the 


\section{Could Tension between Planck's And Other Observations Be Solved?}

non-vacuum solution of the field equations, with inclusion of the gravitational energy momentum tensor (GEMT) and without cosmological constant $\Lambda$, is presented in $(2,3)$. On that way we obtain the solution of the field parameters in the extremally strong gravitational field. In the case of vacuum, the mentioned field parameters are reduced to the well known Schwarzschild vacuum solution of the field equations. Thus, RAF theory is an extension of GRT to the extremely strong gravitational field. Using the related Lagrangean, based on the solution of the line element in a gravitational field we obtain the first form of the cosmological model as function of the gravitational mass $M$ and radius $r$ that has been derived in the reference [39-41]:

$$
\begin{gathered}
\dot{r}= \pm\left[\frac{2 G M}{r}\left(1-\frac{G M}{2 r c^{2}}\right)+\left(\kappa^{2}-\varepsilon^{2}\right) c^{2}\right]^{1 / 2}, \\
\ddot{r}=-\frac{G M}{r^{2}}\left(1-\frac{G M}{r c^{2}}\right) .
\end{gathered}
$$

Here $\kappa$ is the energy conservation constant and $\varepsilon$ is the related Lagrangean parameter. Now applying a mass density $\rho$ and pressure $p$ for an adiabatic process, as well as parameter $\varepsilon=1$ (for time-like geodesics) and spatial curvature constant $\mathcal{K}=$ $\left(1-\kappa^{2}\right)$, the model (2) is transformed into the new cosmological model as function of the mass density $\rho$ and pressure $p$ and gravitational radius $r$ [43]:

$$
\begin{aligned}
& \left(\frac{\dot{r}}{r}\right)^{2}+\frac{\mathcal{K} c^{2}}{r^{2}}=\frac{8 \pi G}{3} \rho\left(1-\frac{2 \pi G}{3} \frac{\rho r^{2}}{c^{2}}\right), \\
& \frac{\ddot{r}}{r}=-\frac{4 \pi G}{3}\left(\rho+\frac{3 p}{c^{2}}\right)\left(1-\frac{4 \pi G}{3} \frac{\rho r^{2}}{c^{2}}\right) .
\end{aligned}
$$

This model is valid for inclusion of the energy momentum tensor, $T_{\mu \eta} \neq 0$ and exclusion of the cosmological constant, $\Lambda$ $=0$.

Comparing the equations (3) with the Friedman equations (1), we can conclude that the gravitational radius $r$ in (3) has the roll of the scale factor $\alpha$ in (1). The first part on the right side of the second equation in (3) states that both the energy density and the pressure cause a deceleration in the expansion of the universe. This is the consequence of gravitation, including that pressure is playing a similar role to that of energy (or mass) density. This is, of course, in accordance with the principles of general relativity. On the other hand, the second part of the right side of the second equation in (3) causes acceleration in the expansion of the universe if $4 \pi G \rho r^{2} / 3 c^{2}>1$ and the deceleration if $4 \pi G \rho r^{2} / 3 c^{2}<1$. In the case that $4 \pi G \rho r^{2} / 3 c^{2}=1$ the acceleration of the universe motion is equal to zero. At this point the acceleration is changed into the deceleration (in the expansion phase) and the deceleration is changed into the acceleration (in the contraction phase). This is the consequence of the non-vacuum solution of the field equations with inclusion of the gravitational energy momentum tensor (GEMT), $T_{\mu \eta}$, and exclusion of the cosmological constant $\Lambda$. Thus, we can see that the new cosmological model (3) generates both repulsive and attractive gravitational forces. In that sense the problem of the dark energy is solved, because the repulsive gravitational energy has the role of the dark energy. Of course, this should be confirmed by the related experiments.

\section{UNIVERSE VELOCITY AND ACCELERATION AT CHARACTERISTIC RADIUSES}

Following the cosmological model $(2,3)$, we can see that the sum of the kinetic energy $E_{k}$ and potential energy $E_{p}$ of a particle with unit mass is equal to constant:

$$
\begin{gathered}
E_{k}=\frac{1}{2}\left[\dot{r}^{2}\right], E_{p}=-\frac{1}{2}\left(\frac{2 G M}{r c^{2}}-\left(\frac{G M}{r c^{2}}\right)^{2}\right), \\
E_{k}+E_{p}=\frac{c^{2}}{2}\left(\kappa^{2}-\varepsilon^{2}\right)=\text { const. }
\end{gathered}
$$

In the case that $\varepsilon=1$ (for time-like geodesics), one obtains the following relations between kinetic and potential energies and the energy conservation constant $\kappa$ :

$$
\begin{gathered}
E_{k}+E_{p}=\frac{c^{2}}{2}\left(\kappa^{2}-1\right), \\
\left|E_{k}\right|=\left|E_{p}\right| \rightarrow \kappa^{2}=1,\left|E_{k}\right|>\left|E_{p}\right| \rightarrow \kappa^{2}>1, \\
\left|E_{k}\right|<\left|E_{p}\right| \rightarrow 0<\kappa^{2}<1 .
\end{gathered}
$$

From the relations (2) and (5) and assuming that the Universe motion follows time-like geodesic $(\varepsilon=1)$, one can derive radial velocity $\dot{r}$ and radial acceleration $\ddot{r}$ of the Universe motion as function of the gravitational mass and radius:

$$
\begin{gathered}
\dot{r}= \pm\left[\frac{2 G M}{r}\left(1-\frac{G M}{2 r c^{2}}\right)+\left(\kappa^{2}-1\right) c^{2}\right]^{1 / 2}, \\
\ddot{r}=-\frac{G M}{r^{2}}\left(1-\frac{G M}{r c^{2}}\right) .
\end{gathered}
$$

The sign (+) in the velocity equation is valid for an expanding phase, while the sign (-) is related to the contracting one. The acceleration equation tells us that the Universe acceleration becomes repulsive if $\left(G M / r c^{2}\right)>1$. One of the conclusions could be that the repulsive gravitational force is the source of dark energy. For the case $\left(G M / r c^{2}\right)=1$ the acceleration is equal to zero and for $\left(G M / r c^{2}\right)<1$ the acceleration is attractive. At the point $\left(G M / r c^{2}\right)=1$, the repulsive acceleration is changing into the attractive one (for expansion phase) and vice versa (for contraction phase).

The velocity equation in (6) has two zeros at the positions $r_{l}$ and $r_{2}$ :

$$
r_{1}=\frac{G M}{(1+\kappa) c^{2}}, \quad r_{2}=\frac{G M}{(1-\kappa) c^{2}}
$$

From the relations (5) and (7) one can see that the hyperspherical (closed) scenario of the Universe motion can be realized only for the case where the energy conservation constant $\kappa$ is satisfying the condition $(0<\kappa<1)$. This means that potential energy is greater than kinetic energy. For that case the velocity equation in (6) has two finite zeros: initial one for $r=r_{1}$, and final one for $r=r_{2}$, see (7). Recent observations of the Planck power spectra prefer that Universe could be closed $[10,11]$. 
Further, the hyperbolic scenario of the universe motion can be realized only for the case where the energy conservation constant $\kappa$ is greater than one $(\kappa>1)$. This means that kinetic energy is greater than potential energy. For that case the velocity relation (6) has only one real zero $r_{1}$ in (7), because the other one gives a negative $r_{2}$. The real zero $r_{1}$ is the initial one for this scenario of the universe motion.

Finally, the flat universe can be realized only for the case where the energy conservation constant $\kappa$ is equal to one ( $\kappa$ $=1$ ). This means that kinetic energy is equal to potential energy. For that case the velocity equation in (6) has one finite initial zero for $r=r_{1}$, and one infinite final zero for $r_{2} \rightarrow$ $\infty$. The initial radius $r_{l}$ of the hyperbolic universe $(\kappa>1)$ is less than the initial radius of the flat universe $(\kappa=1)$ and also less than initial radius of the hyperspherical (closed) universe $(\kappa<1)$.

The maximal radial velocity $\dot{r}_{\max }$ can be obtained from (6) by applying condition that the radial universe acceleration is equal to zero:

$$
\ddot{r}=0 \rightarrow r=r_{c}=\frac{G M}{c^{2}}, \rightarrow \dot{r}_{\max }= \pm \kappa c .
$$

Thus, for $\kappa>1$ the maximal radial velocity is greater than speed of the light in a vacuum. This belongs to the hyperbolic scenario of the Universe motion. For $\kappa=1$ the maximal radial velocity is equal to the speed of the light in a vacuum. This belongs to the flat scenario of the Universe motion. Finally, for $\kappa<1$ the maximal radial velocity is less than speed of the light in a vacuum. This belongs to the hyperspherical (closed) scenario of the Universe motion. Including the previous values of the energy conservation constant $\kappa$ into (8) we obtain the following relation

$$
\dot{r}_{\text {max-hyperbolic }}>\dot{r}_{\text {max-flat }}>\dot{r}_{\text {max-hyperspherical }}
$$

Further, including $r_{1}$ and $r_{2}$ from (7) into the accelerating equation of the universe motion (6), we obtain the related accelerations at the radiuses $r_{1}$ and $r_{2}$ :

$$
\begin{aligned}
& \ddot{r}_{1}=-\frac{G M}{r_{1}^{2}}\left(1-\frac{G M}{r_{1} c^{2}}\right)=\frac{\kappa(1+\kappa)^{2} c^{4}}{G M}, \\
& \ddot{r}_{2}=-\frac{G M}{r_{2}^{2}}\left(1-\frac{G M}{r_{2} c^{2}}\right)=\frac{-\kappa(1-\kappa)^{2} c^{4}}{G M} .
\end{aligned}
$$

The first relation in (10) gives the repulsive initial acceleration $\ddot{r}_{1}$ at the radius $r_{1}$ for all scenarios of the universe motion. On the other side the acceleration $\ddot{r}_{2}$ at radius $r_{2}$ of the universe motion is attractive for hyperspherical and hyperbolic scenarios of the universe motion. Meanwhile, for the flat scenario $(\kappa=1)$ radius $r_{2}$ is going to infinity and the acceleration is going to zero. From the previous consideration we can derive the relation between parameters $\kappa$ and $\mathcal{K}$ :

$$
\begin{gathered}
\left(1-\kappa^{2}\right)=\mathcal{K}, \quad \kappa^{2}=1 \rightarrow \mathcal{K}=0 \rightarrow \text { Flat univ., } \\
\kappa^{2}<1 \rightarrow \mathcal{K}>0 \rightarrow \text { Hyperspherical univ., } \\
\kappa^{2}>1 \rightarrow \mathcal{K}<0 \rightarrow \text { Hyperbolic univ. }
\end{gathered}
$$

Here $\kappa$ is the energy conservation constant derived from the Lagrangean [39] and $\mathcal{K}$ is spatial curvature parameter from the Friedmann equations (1).

For determination of the scenario of the Universe motion given by (11), we have to know the limitations of the energy conservation constant $\kappa$. In that sense, one can start with the radial density relation $\rho_{\mathrm{r}}$ at the minimal and maximal Universe radiuses:

$$
\begin{gathered}
\rho_{r}=\frac{M}{r},\left(\rho_{r}\right)_{\text {max }}=\frac{M}{r_{\text {min }}}=\frac{(1+\kappa) c^{2}}{G}, \\
\left(\rho_{r}\right)_{\text {min }}=\frac{M}{r_{\text {max }}}=\frac{(1-\kappa) c^{2}}{G}, \\
\frac{\left(\rho_{r}\right)_{\text {max }}}{\left(\rho_{r}\right)_{\text {min }}}=\frac{(1+\kappa)}{(1-\kappa)}, \rightarrow \kappa=\frac{\left(\rho_{r}\right)_{\text {max }}-\left(\rho_{r}\right)_{\text {min }}}{\left(\rho_{r}\right)_{\text {max }}+\left(\rho_{r}\right)_{\text {min }}}<1 .
\end{gathered}
$$

From the relations (12) we can see that the limitations of the energy conservation constant are in the region $0<\kappa<1$. This gives the limitation to the spatial curvature of the space $\mathcal{K}>0$. For that case the Universe is a hyperspherical (closed), because flat and hyperbolic universes are excluded by the limitations of parameter $\kappa$ and parameter $\mathcal{K}$ in (11) and (12).

\section{DERIVATION OF HUBbLE PARAMETERS IN RAFT}

Related to the observer, the radius $r(t)$ in (3) has the role of the scale factor $\alpha(t)$ in (1). Thus, the model (3) can be transformed into the following relation:

$$
\begin{aligned}
& \left(\frac{\dot{a}}{a}\right)^{2}+\frac{\mathcal{K} c^{2}}{a^{2}}=\frac{8 \pi G}{3} \rho\left(1-\frac{2 \pi G}{3} \frac{\rho a^{2}}{c^{2}}\right), \\
& \frac{\ddot{a}}{a}=-\frac{4 \pi G}{3}\left(\rho+\frac{3 p}{c^{2}}\right)\left(1-\frac{4 \pi G}{3} \frac{\rho a^{2}}{c^{2}}\right) .
\end{aligned}
$$

Here $a(t)$ and the related time derivations $\dot{\alpha}$ and $\ddot{\alpha}$ have the same meaning as in the Friedman model (1). In order to compare the calculated result of Hubble constant $H_{0}$ for closed Universe (13) with the related observed constant $H_{O}$ for flat $\Lambda$ cold dark matter $(\Lambda \mathrm{CDM})$ cosmological model we started with the observations given by the references $[1,2,3]$ :

$$
\begin{aligned}
& \text { Planck (2018b), } \quad H_{0}=67.4_{-0.5}^{+0.5} \mathrm{kms}^{-1} \mathrm{Mpc}^{-1} \text {, } \\
& D E S+B A O+B B N(2018), H_{0}=67.4_{-1.2}^{+1.1} \mathrm{kms}^{-1} \mathrm{Mpc}^{-1} \text {, } \\
& \text { SHOES( Riess at al.2019), } H_{0}=74.0_{-1.4}^{+1.4} \mathrm{kms}^{-1} \mathrm{Mpc}^{-1} \text {, } \\
& \text { HOLiCOW ( 2019), } \quad H_{0}=73.3_{-1.8}^{+1.7} \mathrm{kms}^{-1} \mathrm{Mpc}^{-1} \text {, } \\
& \mathrm{SHOES}+\mathrm{HOLiCOW}, \quad \mathrm{H}_{0}=73.8_{-1.1}^{+1.1} \mathrm{kms}^{-1} \mathrm{Mpc}^{-1} \text {. }
\end{aligned}
$$

In (14) we have comparison of $H_{0}$ observations for early Universe probes (2018) and late Universe probes (2019). The early Universe probes are related to Planck Collaboration at al. 2018b [3] and combination of clustering and weak lensing 


\section{Could Tension between Planck's And Other Observations Be Solved?}

data, BAO, and big bang nucleo-synthesis, Abbott at al. $2018 b$ [7]. The late Universe probes are related to the results from SHOES (Riess at al. 2019 [6]) and HOLiCOW, 2019 [1]. The latest line in (14) is the combination of two late Universe probes (2019, SHOES and HOLiCOW).

Models (3), (6) and (13) are the cosmological models based on the gravitational parameters only. Therefore, for calculation of the Universe parameters we applied the gravitational model (6), which here is transformed into the cosmological model of the Hubble parameters:

$$
\begin{aligned}
& H^{2}=\left(\frac{\dot{r}}{r}\right)^{2}=\frac{2 G M}{r^{3}}\left(1-\frac{G M}{2 r c^{2}}\right)-\mathcal{K} \frac{c^{2}}{r^{2}}, \\
& \mathcal{K}=(1-\kappa)^{2}, \quad H_{0}=H r_{m p c}, \quad \tau=H^{-1} .
\end{aligned}
$$

This model is more convenient for the application to the gravitational field. In this model $H$ is Hubble parameter, $\mathcal{K}$ is spatial curvature parameter from the Friedmann equations (1), $\kappa$ is the energy conservation constant, $r_{m p c}$ is a radius equal to megaparsec and $\tau$ is time of the Universe expansion from the minimal radius $r_{l}$ (7) to the present radius. Here $\tau$ is calculated from Hubble parameter $H$ as an approximation, instead of using integration of the radial Universe velocity (6) for exact result.

\section{CAlculation of HubBle Parameters for AVERAgE $\mathrm{H}_{0}$ IN EARLY UNIVERSE PROBES}

Here we started with the average value $H_{0}$ from early Universe probes obtained by Planck Collaboration at al. 2018b [3] and Abbott at al. 2018b [7] given in (14):

$$
H_{0}=67.4 \mathrm{kms}^{-1} M p c^{-1} \text {. }
$$

The Hubble's law can be described by the following relation:

$$
\begin{gathered}
H=\frac{v_{m p c}}{r_{m p c}}=\frac{v_{u n}}{r_{u n}}\left(s^{-1}\right), \quad \frac{v_{m p c}}{r_{m p c}}=H_{0}, \\
r_{u n}=\frac{v_{u n}}{v_{m p c} / r_{m p c}}=\frac{v_{u n}}{H_{0}} .
\end{gathered}
$$

Related to the observer, $v_{m p c}$ is the universe expansion velocity at $r_{m p c}$, while $v_{u n}$ is the related universe expansion velocity at the universe radius $r_{u n}$. Following (6) we can see that the maximal radial velocity is at the radius where the radial acceleration is equal to zero, $\ddot{r}=0$. Thus, this radius named as $r_{c}$ and related radial velocity $\dot{r}_{c}$ can be calculated from $(6,8)$ and (11):

$$
\ddot{r}_{c}=0 \rightarrow r=r_{c}=\frac{G M}{c^{2}}, \quad \dot{r}_{c}^{2}=c^{2}(1-\mathcal{K}) .
$$

From the second relation in (18) we can calculate maximal radial velocity for different constants $\mathcal{K}$ :

$$
\begin{gathered}
\dot{r}_{c}^{2}=c^{2}(1-\mathcal{K}) \rightarrow \mathcal{K}=1-\dot{r}_{c}^{2} / c^{2}, \\
\dot{r}_{c}=c \rightarrow \mathcal{K}=0 \rightarrow \text { Flat universe, } \\
\dot{r}_{c}<c \rightarrow \mathcal{K}>0 \rightarrow \text { Closed universe, } \\
\dot{r}_{c}>c \rightarrow \mathcal{K}<0 \rightarrow \text { Hyperbolic universe } .
\end{gathered}
$$

Following the relations (12) and (19) we can see that the Universe is closed $(\mathcal{K}>0)$. This theoretical prediction of RAF theory is in an agreement with the recent observations of the Planck power spectra preferring that the Universe could be closed $[3,11,15]$.

From this prediction and relations (19), we can conclude that the maximal radial expansion velocity in the closed Universe is less than speed of light in vacuum, c. At the moment we do not know the maximal radial velocity of the Universe expansion. Therefore, we started with assumption that the maximal radial velocity in the closed Universe is given by the relation:

$$
v_{u n}=v_{\text {max }}=\dot{r}_{c}=(0.98 c ; 0.95 c ; 0.9 c) .
$$

By the three values in (20) we suppose that the present Universe expansion velocity is close to the speed of the light in vacuum c. Combining the relations (16), (17) and (20) we can calculate the universe radiuses at $r_{c}=r_{u n}$ :

$$
\begin{gathered}
v_{u n}=v_{\max }=\dot{r}_{c}=(0.98 c ; 0.95 c ; 0.9 c), \\
r_{u n}=\frac{v_{u n} \cdot r_{m p c}}{v_{m p c}}=\frac{v_{u n}}{H_{0}}=\frac{v_{u n} \cdot r_{m p c}}{67,4 \cdot 10^{3}}, \\
r_{u n}=r_{c}=(1.317596 ; 1.303871 ; 1.235246) \cdot 10^{26} \mathrm{~m} .
\end{gathered}
$$

The related universe mass can be calculated from the acceleration equation (6), assuming that the acceleration at the radiuses (21) is equal to zero:

$$
\begin{gathered}
\ddot{r}_{c}=-\frac{G M}{r_{c}^{2}}\left(1-\frac{G M}{r_{c} c^{2}}\right)=0 \rightarrow \frac{G M}{r_{c} c^{2}}=1, M=\frac{r_{c} c^{2}}{G}, \\
M_{u n}=(1.774268 ; 1.755786 ; 1.663376) \cdot 10^{53} \mathrm{~kg} .
\end{gathered}
$$

The spatial curvature parameter $\mathcal{K}$ is calculated by using (19) and (20):

$$
\mathcal{K}=1-\frac{\dot{r}_{c}^{2}}{c^{2}}, \dot{r}_{c}=v_{\text {un }} \rightarrow \mathcal{K}=(0.0396 ; 0.0975 ; 0.19) \text {. }
$$

Knowing the spatial curvature parameter $\mathcal{K}(23)$ one can calculate the energy conservation constant $\kappa$ and relations between kinetic and potential gravitational energies in (5):

$$
\begin{gathered}
\mathcal{K}=\left(1-\kappa^{2}\right) \rightarrow \kappa= \pm(1-\mathcal{K})^{1 / 2}, \\
\kappa= \pm(0.999215 ; 0.995235 ; 0.981784), \\
\left(\left|E_{k}\right|<\left|E_{p}\right| ;\left|E_{k}\right|<\left|E_{p}\right| ;\left|E_{k}\right|<\left|E_{p}\right|\right) .
\end{gathered}
$$

Following the relations (16) to (22) and the spatial curvature parameter $\mathcal{K}(23)$, we calculated Hubble constant $H_{0}$ for different maximal radial velocities (20) and related radiuses (21): 


$$
\begin{gathered}
H^{2}=\frac{2 G M}{r^{3}}\left(1-\frac{G M}{2 r c^{2}}\right)-\mathcal{K} \frac{c^{2}}{r^{2}}, H_{0}=H r_{m p c}, \tau=H^{-1}, \\
H=(2.229792 ; 2.1842864 ; 2.1842868) \cdot 10^{-18} \mathrm{~s}^{-1}, \\
H_{0}=(68.804184 ; 67.400025 ; 67.400037) \frac{\mathrm{km}}{\mathrm{s}}, \\
\tau=(14.220964 ; 14.517232 ; 14.517229) \cdot 10^{9} \mathrm{yr} .
\end{gathered}
$$

In (25) $\tau$ is the time from the minimal radiuses (26) to the radiuses given by (21). The minimal and maximal Universe radiuses for the observed Hubble constant (16) are as follows:

$$
\begin{aligned}
& r_{1}=r_{\text {min }}=\frac{G M}{(1+\kappa) c^{2}}, \quad r_{2}=r_{\text {max }}=\frac{G M}{(1-\kappa) c^{2}}, \\
& r_{\text {min }}=(0.659056 ; 0.653492 ; 0.623300) \cdot 10^{26} \mathrm{~m},(26) \\
& r_{\text {max }}=(1.678466 ; 0.273635 ; 0.067811) \cdot 10^{29} \mathrm{~m} .
\end{aligned}
$$

The parameters from (25) that the best correspond to the observed Hubble constant in (16) are as follows:

$$
\begin{gathered}
v_{u n}=\dot{r}_{c}=0.95 c ; \quad r_{u n}=1.303871 \cdot 10^{26} \mathrm{~m}, \\
M_{u n}=1.755786 \cdot 10^{53} \mathrm{~kg} ; \quad H=2.1842864 \cdot 10^{-18} \mathrm{~s}^{-1}, \\
\mathcal{K}=0.0975, \quad \kappa= \pm 0.995235, \quad \tau=14.517232 \cdot 10^{9} \mathrm{yr} ., \\
H_{0}=H \cdot 1 m p c=67.400025 \mathrm{kms}^{-1} \mathrm{Mpc}^{-1} .
\end{gathered}
$$

The parameters from (25) valid for $v_{u n}=\dot{r}_{c}=0.9 c$ are also closed to the observed parameters (16), obtained by Planck Collaboration at al. 2018b [3] and Abbott at al. 2018b [7]. Further, to the present Universe state (27) belong also the related minimal radius, $r_{\text {min }}$, radius $r_{c}$, and maximal radius, $r_{\max }$ :

$$
\begin{gathered}
r_{\text {min }}=6.534923 \cdot 10^{25} \mathrm{~m}, r_{c}=1.303871 \cdot 10^{26} \mathrm{~m}, \\
r_{\text {max }}=0.273635 \cdot 10^{29} \mathrm{~m} .
\end{gathered}
$$

Now we check the condition that radial density $\rho=M / r$ in the region $r<r_{c}$ should be bigger than radial density at $r_{c}$. Also, the radial density in the region $r_{c}$ to $r_{\max }$ should be bigger than radial density at $r_{\max }$. From (12) we derived the following relations:

$$
\begin{gathered}
\left(\rho_{r}\right)_{\text {max }}=\frac{M}{r_{\text {min }}}=\frac{(1+\kappa) c^{2}}{G},\left(\rho_{r}\right)_{\text {min }}=\frac{M}{r_{\text {max }}}=\frac{(1-\kappa) c^{2}}{G}, \\
\left(\rho_{r}\right)_{c}=\frac{M}{r_{c}}=\frac{c^{2}}{G}, \quad 0<\kappa<1, \quad r_{\text {min }}<r_{c}<r_{\text {max }} \rightarrow \\
\left(\rho_{r}\right)_{\text {max }}>\left(\rho_{r}\right)_{c}>\left(\rho_{r}\right)_{\text {min }} .
\end{gathered}
$$

Thus, the radial density propositions are confirmed by the relations (29).

\section{CACUlation of Hubble Parameters For AVERAGE $H_{0}$ IN LATE UNIVERSE PROBES}

In order to compare the previous analyses for the early Universe probes, calculated by the Hubble constant $H_{0}=67.4$ $\mathrm{kms}^{-1} \mathrm{Mpc}^{-1}$, here we use the average value of $H_{0}$ from the late Universe probes obtained by the late results from SHOES (Riess at al. 2019 [6]) and HOLiCOW, 2019 [1]:

$$
H_{0}=73.65 \mathrm{kms}^{-1} M p c^{-1} \text {. }
$$

Combining the relations (30), (17) and (20) we can calculate the universe radiuses at $r_{c}=r_{u n}$ :

$$
\begin{gathered}
v_{u n}=(0.98 c ; 0.95 c ; 0.9 c), \\
r_{u n}=\frac{v_{u n} \cdot r_{m p c}}{v_{m p c}}=\frac{v_{u n}}{H_{0}}=\frac{v_{u n} \cdot r_{m p c}}{73,65 \cdot 10^{3}}, \\
r_{u n}=(1.230905 ; 1.193224 ; 1.130423) \cdot 10^{26} \mathrm{~m} .
\end{gathered}
$$

The related universe mass can be calculated from the acceleration equation (6), assuming that the acceleration at the radiuses (31) is equal to zero:

$$
\begin{gathered}
\ddot{r}_{c}=-\frac{G M}{r_{c}^{2}}\left(1-\frac{G M}{r_{c} c^{2}}\right)=0 \rightarrow \frac{G M}{r_{c} c^{2}}=1, M=\frac{r_{c} c^{2}}{G}, \\
M_{u n}=(1.657531 ; 1.606789 ; 1.522222) \cdot 10^{53} \mathrm{~kg} .
\end{gathered}
$$

The spatial curvature parameters $\mathcal{K}$ and the energy conservation constants $\kappa$ are the same as the values in (23) and (24), respectively. Following the relations (30) to (32) and the spatial curvature parameter $\mathcal{K}(23)$, we calculated Hubble constant $H_{0}$ for different maximal radial velocities and related radiuses (31):

$$
\begin{aligned}
H^{2} & =\frac{2 G M}{r^{3}}\left(1-\frac{G M}{2 r c^{2}}\right)-\mathcal{K} \frac{c^{2}}{r^{2}}, H_{0}=H r_{m p c}, \tau=H^{-1}, \\
H & =(2.3868344 ; 2.3868346 ; 2.3868340) \cdot 10^{-18} s^{-1}, \\
H_{0} & =(73.650003 ; 73.650006 ; 73.649989) \mathrm{kms}^{-1} M p c^{-1}, \\
\tau & =(13.2852915 ; 13.2852908 ; 13.2852938) \cdot 10^{9} \mathrm{yr} .
\end{aligned}
$$

In (33) $\tau$ is the time from the minimal radiuses (34) to the Universe radiuses given by (31). The minimal and maximal Universe radiuses for the calculated Hubble constants (33) are as follows:

$$
\begin{aligned}
& r_{1}=r_{\text {min }}=\frac{G M}{(1+\kappa) c^{2}}, \quad r_{2}=r_{\text {max }}=\frac{G M}{(1-\kappa) c^{2}}, \\
& r_{\text {min }}=(0.615694 ; 0.598036 ; 0.570406) \cdot 10^{26} \mathrm{~m}, \\
& r_{\text {max }}=(1.568032 ; 0.250414 ; 0.313135) \cdot 10^{29} \mathrm{~m} .
\end{aligned}
$$

The parameters from (33) that the best correspond to the observed Hubble constant in (30) are as follows:

$$
\begin{gathered}
v_{u n}=\dot{r}_{c}=0.98 c ; r_{u n}=1.230905 \cdot 10^{26} \mathrm{~m}, \\
M_{u n}=1.657531 \cdot 10^{53} \mathrm{~kg} ; H=2.3868344 \cdot 10^{-18} \mathrm{~s}^{-1}, \\
\mathcal{K}=0.0396, \kappa= \pm 0.999215, \tau=13.2852915 \cdot 10^{9} \mathrm{yr} ., \\
H_{0}=H \cdot 1 \mathrm{mpc}=73.650003 \mathrm{kms}^{-1} \mathrm{Mpc}^{-1} .
\end{gathered}
$$

Further, to the Universe state (35) belong also the related minimal radius, $r_{\text {min }}$, radius $r_{c}$ and maximal radius, $r_{\text {max }}$ : 


$$
\begin{gathered}
r_{\text {min }}=0.615694 \cdot 10^{26} \mathrm{~m}, \\
r_{c}=1.230905 \cdot 10^{26} \mathrm{~m}, \\
r_{\text {max }}=1.568032 \cdot 10^{29} \mathrm{~m} .
\end{gathered}
$$

The parameters from (33) valid for $\dot{v}_{u n}=\dot{r}_{c}=0.95 c$ and $\dot{v}_{u n}=\dot{r}_{c}=0.9 c$ are also close to the observed Hubble parameter (30).

\section{COMPARISON OF PREVIOUS RESUlts}

Here we presented the comparison between the calculated Hubble and cosmological parameters based on the two observed Hubble constant $H_{0}$ in the early and late Universe probes. For calculation of the mentioned parameters we used cosmological gravitational model (15), valid for the closed Universe. Thus, in the first line of the equations (37) we have the calculated result of the Hubble parameter $H$ valid for the early Universe probes (14), $H_{0}=67.4 \mathrm{kms}^{-1} \mathrm{Mpc}^{-1}$. In the second line we have the calculated Hubble parameter $H$ valid for the observed $H_{0}=73.65 \mathrm{kms}^{-1} \mathrm{Mpc}^{-1}$, as the average value obtained from the late Universe probes (14):

$$
\begin{array}{ll}
H_{0}=67.4 k m s^{-1} M p c^{-1}, & H=2.184286 \cdot 10^{-18} s^{-1}, \\
H_{0}=73.65 k m s^{-1} M p c^{-1}, & H=2.386834 \cdot 10^{-18} s^{-1} .
\end{array}
$$

The calculated parameters $H$ belong to the calculated Hubble constants $H_{0 c a l}$ which are the closest to the observed $H_{0}$.

The next relations show the calculated Hubble constants $H_{\text {Ocal }}$ by using Hubble parameters $H$ form (37) and relation $H_{\text {Ocal }}=H r_{m p c}$ :

$$
\begin{aligned}
& H_{0}=67.4 k m s^{-1} M p c^{-1}, H_{0_{c a l}}=67.400025 k \mathrm{~ms}^{-1} M p c^{-1}, \\
& H_{0}=73.65 k m s^{-1} M p c^{-1}, H_{0 c a l}=73.650003 \mathrm{kms}^{-1} M p c^{-1} .
\end{aligned}
$$

From (38) we can see that the smaller difference between observed and calculated Hubble constants $H_{0}$ belongs to the early Universe probes, $H_{0}=67.4 \mathrm{kms}^{-1} \mathrm{Mpc}^{-1}$, see (14).

Now we have comparison between calculated minimal gravitational radiuses that belong to the related observed Hubble constants $H_{0}$ :

$$
\begin{array}{ll}
H_{0}=67.4 \mathrm{kms}^{-1} M p c^{-1}, & r_{\text {min }}=0.653492 \cdot 10^{26} \mathrm{~m}, \\
H_{0}=73.65 \mathrm{kms}^{-1} M p c^{-1}, & r_{\text {min }}=0.615694 \cdot 10^{26} \mathrm{~m} .
\end{array}
$$

From (39) we can see that to the smaller observed Hubble constant $H_{0}=67.4 \mathrm{kms}^{-1} \mathrm{Mpc}^{-1}$ belongs the largest minimal gravitational radius.

It follows the comparison of the calculated Universe radiuses that belong to the observed Hubble constants:

$$
\begin{aligned}
& H_{0}=67.4 \mathrm{kms}^{-1} M p c^{-1}, r_{u n}=1.303871 \cdot 10^{26} \mathrm{~m}, \\
& H_{0}=73.65 \mathrm{kms}^{-1} M p c^{-1}, r_{u n}=1.230905 \cdot 10^{26} \mathrm{~m} .
\end{aligned}
$$

From (40) we also can see that to the smaller observed Hubble constant $H_{0}=67.4 \mathrm{kms}^{-1} \mathrm{Mpc}^{-1}$ belongs the larger calculated Universe radius.
The calculated Universe mass that correspond to the observed Hubble constants are presented in the following relations:

$$
\begin{aligned}
& H_{0}=67.4 k m s^{-1} M p c^{-1}, M_{u n}=1.755786 \cdot 10^{53} \mathrm{~kg}, \\
& H_{0}=73.65 \mathrm{kms}^{-1} M p c^{-1}, M_{u n}=1.657531 \cdot 10^{53} \mathrm{~kg} .
\end{aligned}
$$

From the relations (41) we can see that to the smaller observed Hubble constant $H_{0}=67.4 \mathrm{kms}^{-1} \mathrm{Mpc}^{-1}$ belongs the greater Universe mass.

Finally, we have comparison between the calculated Universe expansion time $\tau$ from the minimal radius (39) to the present Universe radius (40) by using the related calculated Hubble parameter $H$ and the relation $\tau=H^{-1}$, see (15):

$$
\begin{aligned}
& H_{0}=67.4 \mathrm{kms}^{-1} M p c^{-1}, \tau=14.517232 \cdot 10^{9} \mathrm{yr}, \\
& H_{0}=73.65 \mathrm{kms}^{-1} \mathrm{Mpc}^{-1}, \tau=13.285291 \cdot 10^{9} \mathrm{yr} .
\end{aligned}
$$

As we can see from (42), the longer Universe expansion time belongs to the smaller Hubble constant $H_{0}=67.4 \mathrm{kms}^{-1} \mathrm{Mpc}^{-1}$ (Planck (2018b, see (14)). Here $\tau$ is calculated from Hubble parameter $H$ as an approximation, instead of using integration of the radial Universe velocity (6) for the exact result.

\section{DISCUSSION}

The standard $\Lambda$ cold dark matter $(\Lambda \mathrm{CDM})$ cosmological model did not solve the Hubble constant tension between early and late Universe probes. This means that we have to find out the new model that will consistently explain all of the observation data. Here we used new cosmological model based on the Relativistic Alpha Field Theory (RAFT). This model predicts the Closed Universe. Following this cosmological model, we calculated Hubble constants and compared them with the early and late Universe probes. Thus, each probe generates its own cosmological parameters (mass, radius, velocity etc.). This means that the observation methods in the early and late Universe probes are not equivalent each to the others. Therefore, the tension between the early (Planck's) and late Universe probes could not be solved.

\section{REFERENCES}

[1] Wong, K.C., et al. HOLiCOW XIII. A $2.4 \%$ measurement of $H_{0}$ from lensed quasar: $5.3 \sigma$ tension between early and late-Universe probes (2019). arXiv:1907.04869v2 [astro-ph.CO] 6 Nov 2019.

[2] Planck Collaboration at al., 2018a, preprint (arXiv:1807.06205).

[3] Aghanim,N. at. al. Planck Collaboration at al., 2018b, preprint (arXiv:1807.06209).

[4] Weinberg, D.H., Mortonson, M.J., Eisenstein, D.J., Hirata, C., Ries, A.G. \& Rozo, E., (2013), Phys. Rep., 530, 87.

[5] Sandage, A., Tammann, G.A., Saha, A., Reindl, B., Macchetto, F.D., \& Panagia, N., (2006), ApJ, 653, 843.

[6] Riess, A.G., et al., (2019). ApJ, 876, 85.

[7] Abbott, T.M.C., et al., (2018b), MNRAS, 480, 3879.

[8] Abbott, T.M.C., et al., (2018a), Phys. Rev. D, 98, $043526 .$.

[9] Abbott, B.P., et al., (2017), Nature, 551, 85.

[10] Di Valentino, E., Melchiorri, A. \& Silk J. Plank evidence for a closed Universe and a possible crisis for cosmology. Nat. Astronomy (2018). https://doi.org/10.1038/s41550-019-0906-9

[11] Di Valentino, E. Shape of Universe: could it be curved, not flat? Jamaica Gleaner (2019). http://jamaica-gleaner.com/authors/elenora-di-valentino-contributor

[12] Stephen M.F., et. al., Prospects for resolving the Hubble constant 
tension with standard sirens (2018). arXiv:1802.03404v2 [astro-ph.CO] 21 May 2018.

[13] Rui Y.G., Jing F.Z., Xin Z., Can the $\mathrm{H}_{0}$ tension be resolved in extension to $\Lambda$ CDM cosmology? (2019), Journal of Cosmology and Astroparticle Physics, Volumen 2019, February 2019.

[14] Ashtekar A., Gupt B., Jeong D., Sreenath V., Alleviating the tension in CMB using Planck-scale Physics (2020). arXiv:2001.11689v1 [astro-ph.CO] 31 Jan 2020.

[15] Friedman, A. On the possibility of a world with constant negative curvature of space, General Relativity and Gravitation 31 (1999) 2001-2008.

[16] Lemaitre, G. Expansion of the universe, a homogeneous universe of constant mass and increasing radius accounting for the radial velocity of extra galactic nebulae, Monthly Notices of the Royal Astronomical Society 91 (1921) 483-490.

[17] Robertson, H.P. Kinematics and world structure, Astrophysical Journal 82 (1935) 284-301.

[18] Robertson, H.P. Kinematics and world structure II, Astrophysical Journal 83 (1936) 187-201.

[19] Robertson, H.P. Kinematics and world structure III, Astrophysical Journal 83 (1936) 257-271.

[20] Walker, A. G. On Milne's theory of world structure. Proceedings of the London Mathematical Society 242 (1937) 90-127.

[21] Lachieze-Rey, M., Luminet, J.P. Cosmic topology, Physical Reports 254 (1995) 135-214.

[22] Perlmutter S. et al., Nature 391 (1998) 51; Riess A. et al., Astron. J. 116 (1998) 1009

[23] Novakovic, B.M., Novakovic, D., Novakovic, A. Cosmological constant problem solution valid for both Planck's and cosmological scales. AIP Conference Proceedings 839 (2006) 144. https://doi.org/10.1063/1.2216624

[24] Kremer, G.M. Cosmological models described by a mixture of der Waals fluid and Dark energy, Phys. Rev. D 68 (2003) 123507.

[25] Jorás, S.E., Stuchi, T.J. Chaos in a closed Friedmann Robertson Walker universe: An imaginary approach, Phys. Rev. D 68 (2003) 123525.

[26] Susperregi, M. Dark energy and Dark matter from an inhomogeneous dilaton, Phys. Rev. D 68 (2003) 123509.

[27] Castro, P.G., Douspis,M., Ferreira, P.G. Scale of homogeneity of the universe from WMAP, Phys. Rev. D 68 (2003) 127301.

[28] Avelino, P.P., Martins, C. J.A.P., Santos, C., Shellard, E.P.S. Topological defects: A problem for cyclic universe, Phys. Rev. D 68 (2003) 123502.

[29] Khoury, J., Steinhardt, P.J., Turok, N. Designing cyclic universe models, Phys. Rev. Lett. 92 (2004) 031302.

[30] Lidsey, J.E. Physical Review D 70 (2004) 041302.

[31] Clifton, T., Clarkson, C., Bull, P. Isotropic blackbody: Cosnhir] microwave background radiation as evidence for a homogeneous universe, Phys. Rev. Lett. 109 (2012) 051303.

[32] Lincoln, M., Wasser, A. Spontaneous creation of the Universe Ex Nihilo, Physics of the Dark Universe 2 (2013) 195-199. https://doi.org/10.1016/j.dark.2013.11.004

[33] Baldi, M. Dark Energy simulations, Dark Universe 1 (2012) 162-193. https://doi.org/10.1016/j.dark.2012.10.004

[34] Kuhlen, M., Vogelsberger, M., Angulo, R. Numerical simulations of the dark universe: State of the art and the next decade, Dark Universe 1 (2012) 50-93. https://doi.org/10.1016/j.dark.2012.10.002

[35] Huang, P., Yuan, F.F. Uniformly expanding vacuum: A possible interpretation of the dark energy, Physics of the Dark Universe 12 (2016) 45-49

[36] Ghaffari, S., Moradpour , H., Bezerra, V. B., MoraisGraça, J.P. Lobo, I.P. Tsallis holographic dark energy in the brane cosmology. https://doi.org/10.1016/j.dark.2018.11.007

[37] Marttens, R. von, Casarini, L., Mota, D.F., Zimdahl, W. Cosmological constraints on parametrized interacting dark energy. https://doi.org/10.1016/j.dark.2018.10.007

[38] Casalino, A., Rinaldi, M., Sebastiani, L., Vagnozzi, S. Mimicking dark matter and dark energy in a mimetic model compatible with GW170817, Physics of the Dark Universe 22 (2018) 108-115. https://doi.org/10.1016/j.dark.2018.10.001

[39] Novakovic, B. M.: Relativistic alpha field theory - Part I: Determination of Field Parameters. International Journal of New Technology and Research (IJNTR) ISSN:2454-4116, 1, 5 (2015)23-30. https://doi.org/10.31871/IJNTR.1.5.15

[40] Novakovic, B. M.: Relativistic alpha field theory-Part II: Does a Gravitational Field Could be Without Singularity? International
Journal of New Technology and Research (IJNTR) (2015). https://doi.org/10.31871/IJNTR.1.5.16

[41] Novakovic, B. M.: Relativistic alpha field theory-Part III: Does Gravitational Force Becomes Positive if $\left(\mathrm{GM} / \mathrm{rc}^{2}\right)>1$ ? International Journal of New Technology and Research (IJNTR) (2015). https://doi.org/10.31871/IJNTR.1.5.17

[42] Novakovic, B.M. Physical Properties of Energy-Momentum Tensor of Gravitational Field in RAF Theory, International Journal of New Technology and Research (IJNTR) ISSN:2454-4116, 4, 11 (2018) 19-25. https://doi.org/10.31871/IJNTR.4.11.10

[43] Novakovic, B.M. Is positive Gravitational Force Source of Dark Energy?, International Journal of New Technology and Research (IJNTR) ISSN:2454-4116, 1, 7 (2015) 6-13. https://doi.org/10.31871/IJNTR.1.7.5

\section{ACKNOLIEDGEMENTS}

This work was supported by the National Scientific Foundation of Republic of Croatia (grant number: 120-1201842-3048).

\section{COMPETING INTERESTS}

The author declares no competing interests.

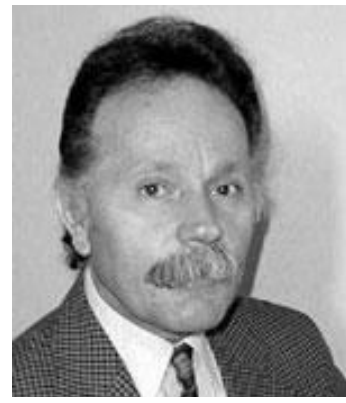

Branko Novakovic is a Professor emeritus at FSB - University of Zagreb, Croatia. Prof. Novakovic received his $\mathrm{PhD}$ from the University of Zagreb in 1978. His research of interest includes physics, control systems, robotics, neural networks, and fuzzy control. He is author of three books; Relativistic Alpha Field Theory (RAFT, e-book, 2016), Control Methods in Robotics, Flexible Manufacturing Systems and Processes (1990), Control Systems (1985) and the first co-author of a book Artificial Neural Networks (1998). He has published over 240 research papers in his research of interest. 